\title{
Reduced meat consumption and its effect on serum lipid profile; an intervention trial in healthy volunteers
}

\author{
E.J. Simpson ${ }^{1}$, M. Clark ${ }^{2}$, A. Razak ${ }^{1}$ and A. Salter ${ }^{2}$ \\ University of Nottingham Schools of ${ }^{1}$ Life Sciences, Medical School, Queens Medical Centre, Nottingham, NG72UH, \\ $U K$ and \\ ${ }^{2}$ Biosciences, Sutton Bonington Campus, Loughborough, LE12 5RD UK
}

Meat represents an important part of a healthy diet for many adults across the globe, supplying high quality protein and micronutrients ${ }^{(1)}$. The bioavailability and concentration of these micronutrients in plant-derived foods are generally lower ${ }^{(1)}$, and plant protein is often incomplete. However, high red and processed meat intake is implicated in the development of cardiovascular disease and certain cancers ${ }^{(2)}$ (in particular colorectal cancer ${ }^{(3)}$ )_ENREF_11, and globally, livestock farming has been recognised as having a negative environmental impact ${ }^{(4)}{ }_{\text {ENREF_1 }}$ EN.

The current study aimed to reduce the red and processed meat intake of healthy, non-obese $\left(18-28 \mathrm{~kg} / \mathrm{m}^{2}\right)$ omnivores by $50 \%$, and to monitor any changes to cardiovascular risk factors that occurred as a result of this behaviour change. A non-randomised, singlegroup study design, comprising an initial 4-wk control period, followed by a 12-wk intervention, was used. Thirty-seven men and women $(21-48 \mathrm{y} ; 16 \mathrm{M}: 21 \mathrm{~F})$, who ate $\geqslant 4$ portions/wk of red and processed meat (with $\geqslant 3$ portions being consumed as main meals), were assessed before (V1) and after (V2) the control period and before (V2), at wk 6 (V3) and at the end of intervention (V4). Dietary intake was estimated using 4-day diet diaries in the week prior to attending each visit. During the intervention, participants were given information regarding how meat intake could be safely reduced in their diet, including a recipe book containing ideas for meals using meat-free or reduced-meat products. In addition, a range of these foodstuffs were provided to participants to assist with meat-reduction if they wished to use them. The study was approved by the University of Nottingham Medical School committee and conducted according to the guidelines laid down in the Declaration of Helsinki.

Compared with V1, mean protein intake from red and processed meat was reduced by $67 \%$ at V3 and $47 \%$ at V4. Compared with the control period, total dietary protein and fat intake in females was lower during the intervention (partial eta ${ }^{2} 0 \cdot 283, \mathrm{P}<0.05$ and partial eta ${ }^{2} 0.502, \mathrm{P}=0.001$ respectively), with males showing no change in protein but a trend for lower fat intake (partial eta ${ }^{2} 0.211$, $\mathrm{P}=0.064)$. BMI in both genders remained stable over the study. Mean total cholesterol (TC), LDL and HDL concentration was lower in males at V4 compared with V2 (effect size $-0.52,-0.41$ and $-0.15 \mathrm{mmol} / 1$, respectively; each $\mathrm{P}<0.01$ ), although there was no change in Total: HDL ratio (effect size $0.03, \mathrm{P}=0.757$ ). In males, TC and LDL concentration at $\mathrm{V} 2$ was negatively correlated with the change in these variables at V4 $\left(\mathrm{R}^{2}=0.647\right.$ and $\mathrm{R}^{2}=0.595$, respectively; both $\left.\mathrm{P}<0.001\right)$. No changes to serum lipids were observed in females.

It was possible for omnivores to reduce their red and processed meat intake over a period of 12-weeks. Although males demonstrated serum TC and LDL levels below concentrations which would require therapeutic intervention, this dietary change reduced TC and LDL in these individuals. Moreover, in males, reduction in red and processed meat intake appeared to confer greatest benefit to those who had higher TC and LDL levels at the start.

1. Biesalski HK (2005) Meat as a component of a healthy diet - are there any risks or benefits if meat is avoided in the diet? Meat science 70, 509-524

2. Rohrmann S, Overvad K, Bueno-de-Mesquita HB et al. (2013) Meat consumption and mortality-results from the European Prospective Investigation into Cancer and Nutrition. BMC medicine 11, 63.

3. Bouvard V, Loomis D, Guyton KZ et al. (2015) Carcinogenicity of consumption of red and processed meat. The Lancet Oncology 16, 1599-1600.

4. Steinfeld H, Gerber P, Wassenaar T et al. (2006) Livestock's Long Shadow; environmental issues and options. Rome: Food and Agriculture Organization of the United Nations. 